\title{
On $\mathrm{BCL}^{+}$-Algebras
}

\author{
Yonghong Liu \\ School of Automation, Wuhan University of Technology, Wuhan, China \\ Email: hylinin@163.com
}

Received October 11, 2011; revised November 24, 2011; accepted December 5, 2011

\begin{abstract}
This paper presents the $\mathrm{BCL}^{+}$-algebras, which is derived the fundamental properties. Results are generalized with version of BCL-algebras [5], using some unusual for a binary relation * and a constant 1 (one) in a non-empty set $X$, one may take different axiom systems for $\mathrm{BCL}^{+}$-algebras.
\end{abstract}

Keywords: BCL-Algebra; $\mathrm{BCL}^{+}-$Algebra; Logic Algebra

\section{Introduction}

The BCK/BCI/BCH-algebra (see [1-4]) has been a major issue, but BCL-algebra (see [5]) is a new algebra structure - and we started to grasp the properties. This paper presents the $\mathrm{BCL}^{+}$-algebras, we show that under our formulation, the $\mathrm{BCL}^{+}$-algebra is a variant of a $\mathrm{BCL}-$ algebra. We can define by taking some axioms and important properties in this way for the $\mathrm{BCL}^{+}$-algebras.

A BCL-algebra may be defined as a non-empty set $X$ with a binary relation * and a constant 0 (zero) satisfying the following axioms:

Definition 1.1. [5] An algebra $(X ; *, 0)$ of type $(2,0)$ is said to be a BCL-algebra if and only if for any $x, y, z \in X$, the following conditions:

1) BCL-1: $\quad x * X=0$;

2) BCL-2: $x * y=0$ and $y * x=0$ imply $x=y$;

3) BCL-3:

$$
(((x * y) * z) *((x * z) * y)) *((z * y) * x)=0 .
$$

Such set $X$ in Definition 1.1 is called the underlying set of a BCL-algebra $(X ; *, 0)$, which needs the following theorem:

Theorem 1.1. [5] Algebra $(X ; *, 0)$ of type $(2,0)$ is a BCL-algebra if and only if it satisfies the following conditions: for all $x, y, z \in X$,

1) BCL-1: $\quad x * x=0$;

2) BCL-2: $\quad x * y=0$ and $y * x=0$ imply $x=y$;

3) $((x * y) * z) *((x * z) * y)=(z * y) * x$.

\section{Main Result}

The $\mathrm{BCL}^{+}$product, denoted by *. We call the binary operation * on $X$ the * product on $X$, and the constant 1 (one) of $X$ the unit element of $X$. For brevity we often write $X$ instead of $(X ; *, 1)$. We begin with the following defini- tion:

Definition 2.1. An algebra $(X ; *, 1)$ is called a $\mathrm{BCL}^{+}-$ algebra if it satisfies the following laws hold: for any $x, y, z \in X$,

1) $\mathrm{BCL}^{+}-1: \quad x * x=1$;

2) $\mathrm{BCL}^{+}-2: x * y=1$ and $y * x=1$ imply $x=y$;

3) $\mathrm{BCL}^{+}-3$ :

$$
((x * y) * z) *((x * z) * y)=(z * y) * x .
$$

Such definition, clearly, the $\mathrm{BCL}^{+}$-algebra is a generalization of the BCL-algebra, imply a BCL-algebra is a $\mathrm{BCL}^{+}$-algebra, however, the converse is not true. We illustrate with the next theorem.

Theorem 2.1. A $\mathrm{BCL}^{+}$-algebra is existent.

Proof. The proof of this Theorem 2.1 is not difficult and uses only example. Let $X=\{0,1,2,3\}$. Define an operation * on $X$, which are given in Table 1.

Then $(X ; *, 1)$ is a proper $\mathrm{BCL}^{+}$-algebra. It is easy to verify that there are

BCI-1:

$$
\begin{aligned}
& ((2 * 3) *(2 * 1)) *(1 * 3) \\
& =(1 * 1) * 3 \\
& =1 * 3 \\
& =3 \neq 0 ;
\end{aligned}
$$

BCI-2:

$$
\begin{aligned}
& (2 *(2 * 3)) * 3 \\
& =(2 * 1) * 3 \\
& =1 * 3 \\
& =3 \neq 0 ;
\end{aligned}
$$

BCH-3: 1) The left side of the equation is

$$
(2 * 3) * 1=1 * 1=1 \text {; }
$$


Table 1. $\mathrm{BCL}^{+}$operation.

\begin{tabular}{lllll}
\hline $\boldsymbol{*}$ & $\mathbf{0}$ & $\mathbf{1}$ & $\mathbf{2}$ & $\mathbf{3}$ \\
\hline $\mathbf{0}$ & 1 & 0 & 0 & 0 \\
$\mathbf{1}$ & 0 & 1 & 3 & 3 \\
$\mathbf{2}$ & 3 & 1 & 1 & 1 \\
$\mathbf{3}$ & 1 & 3 & 3 & 1 \\
\hline
\end{tabular}

2) The right side of the equation is

$$
(2 * 1) * 3=1 * 3=3 \text {. }
$$

In the expression we see that $1 \neq 3$.

BCL-3:

$$
\begin{aligned}
& (((2 * 3) * 1) *((2 * 1) * 3)) *((1 * 3) * 2) \\
& =((1 * 1) *(1 * 3)) *(3 * 2) \\
& =(1 * 3) * 3 \\
& =3 * 3 \\
& =1 \neq 0 .
\end{aligned}
$$

$\left.\mathrm{BCL}^{+}-3: 1\right)$ The left side of the equation is

$$
\begin{aligned}
& ((2 * 3) * 1) *((2 * 1) * 3) \\
& =(1 * 1) *(1 * 3) \\
& =1 * 3 \\
& =3
\end{aligned}
$$

2) The right side of the equation is

$$
(1 * 3) * 2=3 * 2=3 \text {. }
$$

In the expression we see that $\mathrm{BCL}^{+}-3$ is valid. In fact, it is not difficult to verity that $\mathrm{BCL}^{+}-1$ and $\mathrm{BCL}^{+}-2$ are valid.

A $\mathrm{BCL}^{+}$-algebra $(X ; *, 1)$ is a partially ordered relation $\leq$ on $X$, now we obtain the following definition:

Definition 2.2. Suppose that $(X ; *, 1)$ is a $\mathrm{BCL}^{+}$-algebra, the ordered relation if

$$
\begin{gathered}
x \leq y \text { if and only if } x * y=1, \\
\text { for all } x, y \in X,
\end{gathered}
$$

then $(X ; \leq)$ is partially ordered set and $(X ; *, 1)$ is an algebra of partially ordered relation.

Corollary 2.1. Let every $x \in X$. Then 1 (one) is maximal element in a $\mathrm{BCL}^{+}$-algebra $(X ; *, 1)$ such that

$$
1 \leq x \text { imply } x=1 \text {. }
$$

Definition 2.3. A $\mathrm{BCL}^{+}$-algebra $X$ is called proper $\mathrm{BCL}^{+}$-algebra if $X$ is not a BCL-algebra.

Example 2.1. Let $X=\{0, a, b, c, 1\}$. We define an operation * on $X$ by Table 2 .

In fact, it is not difficult to verify that $(X ; *, 1)$ is a $\mathrm{BCL}^{+}$-algebra.
Table 2. BCL ${ }^{+}$operation.

\begin{tabular}{cccccc}
\hline$*$ & $\mathbf{0}$ & $\boldsymbol{a}$ & $\boldsymbol{b}$ & $\boldsymbol{c}$ & $\mathbf{1}$ \\
\hline $\mathbf{0}$ & 1 & 0 & 0 & 0 & 0 \\
$\boldsymbol{a}$ & $a$ & 1 & 1 & $c$ & 1 \\
$\boldsymbol{b}$ & $b$ & $a$ & 1 & $c$ & 1 \\
$\boldsymbol{c}$ & $c$ & $b$ & $c$ & 1 & 1 \\
$\mathbf{1}$ & 0 & 1 & 1 & 1 & 1 \\
\hline
\end{tabular}

Theorem 2.2. Assume that $(X ; *, 1)$ is any a $\mathrm{BCL}^{+}$algebra. Then the following hold: for any $x, y, z \in X$,

1) $(x *(x * y)) * y=1$;

2) $x * 1=x$ imply $x=1$;

3) $((x * y) *(x * z)) *(z * y)=1$;

4) $\mathrm{BCL}^{+}-2: \quad x * y=1$ and $y * x=1$ imply $x=y$.

Proof. Necessity. By $\mathrm{BCL}^{+}-1$ and 3), we obtain

$$
(x *(x * y)) * y=((x * 1) *(x * y)) *(y * 1)=1 .
$$

So, 1) holding.

By the same reasons, we derive

$$
x * 1=(x * 1) * 1=((x * 1) *(x * 1)) *(1 * 1)=1 .
$$

Hence, 2) holding.

Sufficiency. It only needs to show $\mathrm{BCL}^{+}-1$. Substituting $y$ for 1 (one) in 1), we have

$$
(x *(x * 1)) * 1=1 .
$$

Replacing $x * 1$ by $y$ and $x$ by $z$ in 3 ), it follows

$$
((x *(x * 1)) *(x * x)) *(x *(x * 1))=1 .
$$

Using 2) and $\mathrm{BCL}^{+}-1$, we get

$$
((x *(x * 1)) * 1) *(x *(x * 1))=1
$$

Clearly, an application of (2.5) to (2.7) can give

$$
1 *(x *(x * 1))=1 .
$$

Comparing (2.4) with (2.7) and using $\mathrm{BCL}^{+}-2$, we get

$$
x *(x * 1)=1 .
$$

Also, by 2) and 1), the following holds:

$$
(x * 1) * x=(x *(x * x)) * x=1 .
$$

Combining (2.9) and (2.10) with 4) create $x * 1=x$. So Theorem 2.2 is valid.

Theorem 2.3. An algebra $(X ; *, 1)$ is a $\mathrm{BCL}^{+}$-algebra if and only if it satisfies the following conditions: for all $x, y, z \in X$,

1) $\mathrm{BCL}^{+}-1: \quad x * X=1$;

2) $\mathrm{BCL}^{+}-2: x * y=1$ and $y * x=1$ imply $x=y$; 
3) $(((x * y) * z) *((x * z) * y)) *((z * y) * x)=1$;

4) $x *(1 * y)=x$.

Proof. The proof is routine. Necessity. To prove 1). By $\mathrm{BCL}^{+}-3$.

$$
\begin{aligned}
x * x & =(x * x) * 1 \\
& =((1 * x) * x) *((1 * x) * x)=1 .
\end{aligned}
$$

Then 1) holding.

Sufficiency. Substituting $x * 1$ for $y$ and $x$ for $z$ in 3), by $\mathrm{BCL}^{+}-3$ and 1$)$, it follows

$$
\begin{aligned}
& (((x *(x * 1)) * x) *((x * x) *(x * 1))) *((x *(x * 1)) * x) \\
& =((1 * x) *(1 * x)) *(1 * x) .
\end{aligned}
$$

Also, substituting $1 * x$ for $x$ in $(2.11)$, by $\mathrm{BCL}^{+}-3$ and 1), we have

$$
\begin{aligned}
& ((1 * x) *(1 * x)) *(1 * x) \\
& =((x * x) * x) *((x * x) * x) \\
& =(1 * x) *(1 * x)=x * x=1 .
\end{aligned}
$$

Using Theorem 2.2 with 4), we obtain

$$
x * 1=x *(1 * x)=x .
$$

Hence $(X ; *, 1)$ is a $\mathrm{BCL}^{+}$-algebra.

\section{REFERENCES}

[1] Y. Imai and K. Iséki, "On Axiom System of Propositional Calculi XIV," Proceedings of the Japan Academy, Vol. 42, No. 1, 1966, pp. 19-22. doi:10.3792/pja/1195522169

[2] K. Iséki, "An Algebra Related with a Propositional Calculus," Proceedings of the Japan Academy, Vol. 42, No. 1, 1966, pp. 26-29. doi:10.3792/pja/1195522171

[3] K. Iseki, "On BCI-Algebras," Mathematics Seminar Notes (Kobe University), Vol. 8, No. 1, 1980, pp. 125-130.

[4] Q. P. Hu and X. Li, "On BCH-Algebras," Mathematics Seminar Notes (Kobe University), Vol. 11, No. 2, 1983, pp. 313-320.

[5] Y. H. Liu, "A New Branch of the Pure Algebra: BCLAlgebras," Advances in Pure Mathematics, Vol. 1, No. 5, 2011, pp. 297-299. doi:10.4236/apm.2011.15054 